\title{
The pattern of intramural veins of the left ventricle of the human heart ${ }^{1}$
}

\author{
Geoffrey Farrer-Brown and M. H. Tarbit \\ From the Bland-Sutton Institute of Pathology, Middlesex Hospital Medical School, London
}

A detailed injection, microradiographic, and histological necropsy study of 18 hearts showed that the pattern of myocardial veins differed from the arterial pattern. In 'normal' hearts, large drainage veins began in the subendocardial zone and coursed fairly directly towards the epicardium, maintaining a comparatively even calibre throughout. Smaller, but similar, drainage veins also began in the middle of the myocardial wall. In the outer myocardium small groups of these vessels converged to form a single vein which then entered, almost at right angles, the pericardial veins. In general, small veins within the myocardium entered directly into the large drainage veins without any extensive intermediate sized venous network. In 'abnormal' hearts with left ventricular hypertrophy the myocardial veins appeared basically normal but were more widely separated and consequently overall less dense on microradiography. Interruption of main drainage veins and loss of normal pattern were seen in areas of myocardial fibrosis associated with coronary artery disease. In a heart with severe generalized coronary artery atheroma the normal pattern in the inner half of the wall was replaced by a 'plexus' of small veins in which the majority of vessels coursed circumferentially. The possible significance of both the normal and abnormal vein pattern is discussed.

Knowledge of the pattern of the intramural veins of the human heart is limited. Though many postmortem injection studies have been made of the coronary arteries few workers have investigated the patterns of the veins and no study of the fine details of the intramural veins in normal and diseased hearts appears to have been carried out.

Truex and Angulo (1952) stated that there was a 'veritable feltwork of smaller and smaller venous radicles within the myocardium of the left ventricle' but illustrated this appearance only on whole heart $x$-rays. Parsonnet (1953) demonstrated veins only in the outer two-thirds of the myocardium on $x$-rays of serial sections of the ventricles after injection of a barium sulphate - agar medium. More recently Baroldi and Scomazzoni (1967) using 'geon' and 'neoprene' latices injection media followed by corrosion of the myocardium described, but did not illustrate, a fine network of veins extending throughout the whole thickness of the wall.

This lack of knowledge of the pattern of the intramural veins is surprising considering both the important physiological role of the venules and the Received 25 June 1974.

1 This work was supported by a grant from the British Heart Foundation. need for further information concerning the drainage of blood from a scarred myocardium, when the haemodynamics of coronary blood flow is studied.

Consequently the present authors have extended their necropsy studies of the arterial system of the human heart (Farrer-Brown, 1967, 1968a, b, c, d; Farrer-Brown and Rowles, 1969; Farrer-Brown and Tarbit, 1973a, b) to include the venous system. The present paper reports the appearance of the intramural vein pattern of the left ventricle in a preliminary study of normal and abnormal human hearts.

\section{Method}

The basic technique has been described previously (Farrer-Brown, 1968a) but a few minor modifications were used in this study and briefly it is as follows. Radio-opaque medium (Colorpaque, Pilot Chemical Ltd. or Micropaque, Damancy \& Co.), with 4 per cent added gelatin was injected down the coronary sinus at a pressure of 80 to $100 \mathrm{mmHg}$. After fixation the hearts were sectioned on a bacon slicer into uniform $5 \mathrm{~mm}$ thick slices from the apex up to just below the mitral valve. The overall vascular pattern of these slices was visualized by $x$-raying on to fine grain film (Microtex, Kodak), using a water-cooled low voltage Machlett $x$-ray tube: $25 \mathrm{kV}$ and $20 \mathrm{~mA}$ exposures were used for to 
TABLE Details of patients

\begin{tabular}{|c|c|c|c|c|c|}
\hline $\begin{array}{l}\text { Case } \\
\text { No. }\end{array}$ & Age $(y r)$ & Sex & $\begin{array}{l}\text { Heart } \\
\text { weight }(g)\end{array}$ & $\begin{array}{l}\text { WHO grade } \\
\text { coronary artery } \\
\text { atheroma }\end{array}$ & Main finding at necropsy \\
\hline \multicolumn{6}{|c|}{ 'Normal' hearts } \\
\hline I & 37 & $\mathbf{F}$ & 275 & $0-1$ & Cerebral tumour \\
\hline 3 & 62 & $\mathbf{M}$ & 310 & $\mathbf{I}$ & Cerebral tumour \\
\hline 4 & 23 & $\mathbf{M}$ & 330 & $\mathbf{I}$ & Encephalitis \\
\hline 5 & 33 & $\mathbf{F}$ & 305 & $0-1$ & Multiple injuries \\
\hline 6 & 75 & $\mathbf{F}$ & 210 & I & Carcinomatosis; primary site unknown \\
\hline 7 & 70 & $\mathbf{F}$ & 345 & $\mathbf{I}$ & Carcinoma of lung \\
\hline 9 & 29 & $\mathbf{M}$ & 330 & $\mathbf{I}$ & Multiple injuries \\
\hline Io & 62 & $\mathbf{F}$ & 275 & $0-1$ & Carcinoma of breast \\
\hline 13 & 58 & $\mathbf{F}$ & 300 & $\mathbf{I}$ & Carcinoma of breast \\
\hline I5 & 21 & $\mathbf{M}$ & 355 & 0 & Carcinoma of thyroid \\
\hline 16 & 53 & $\mathbf{F}$ & 175 & $\mathbf{I}$ & Carcinoma of breast \\
\hline 17 & 53 & $\mathbf{F}$ & 285 & $0-\mathbf{r}$ & Carcinoma of oesophagus \\
\hline \multicolumn{6}{|c|}{ Abnormal hearts } \\
\hline 2 & 72 & $\mathbf{M}$ & 505 & 3 & $\begin{array}{l}\text { Hypertension, chronic bonchitis and emphysema, } \\
\text { pulmonary embolus }\end{array}$ \\
\hline 8 & 60 & $\mathbf{M}$ & 610 & 2 & Cerebrovascular accident; hypertension \\
\hline II & 6I & $\mathbf{M}$ & 470 & $\overline{\mathbf{I}}$ & Carcinoma of adrenal; hypertension \\
\hline 12 & 53 & $\mathbf{M}$ & 480 & 3 & Ruptured abdominal aortic aneurysm \\
\hline 14 & 62 & $\mathbf{F}$ & 465 & 2 & $\begin{array}{l}\text { Hypertensive heart disease; cerebrovascular acci- } \\
\text { dent }\end{array}$ \\
\hline 18 & 46 & $M$ & 380 & 2 & Diabetes mellitus; bronchopneumonia \\
\hline
\end{tabular}

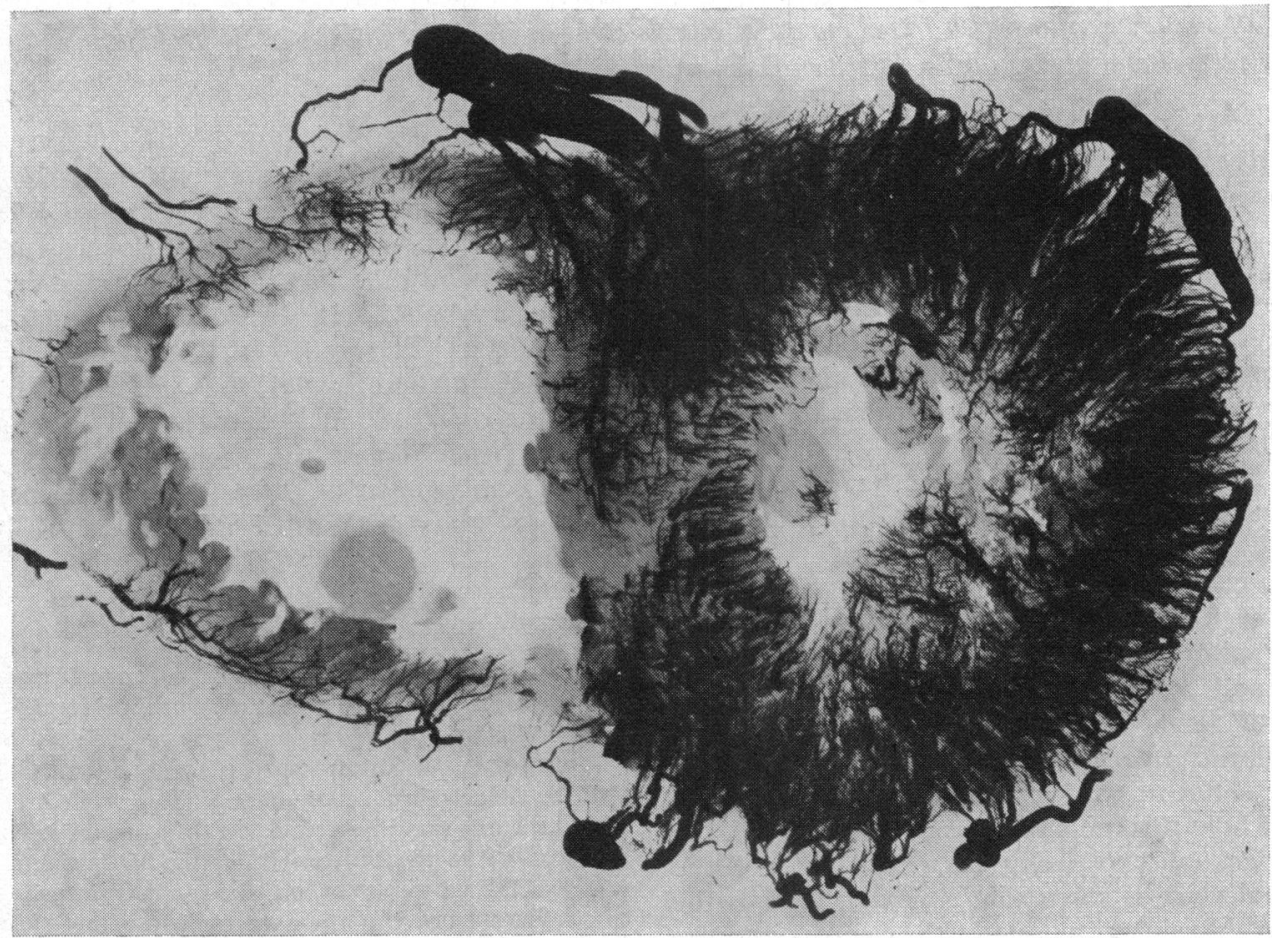

FIG. I Microradiograph of a $5 \mathrm{~mm}$ transverse ventricular slice of the $310 \mathrm{~g}$ 'normal' heart from a 62-year-old man who died of a cerebral tumour. The density of veins in the left ventricle is greater than that seen with arterial injections. $(\times 2$. 
seconds, with a tube distance of $64 \mathrm{~cm}$. A similar exposure, but for 20 minutes, was used to $x$-ray the atrial portions of the hearts, submerged under water, on to Crystallex film (Kodak). To demonstrate the finest vessels, selected ventricular slices were radiographed on Kodak maximum Resolution plates using the same $x$-ray tube and exposures of $25 \mathrm{kV}$ and 10 to $20 \mathrm{~mA}$ for 15 to 30 minutes according to the thickness of the slice. The plates were developed for 4 minutes at $68^{\circ} \mathrm{C}$ in high contrast developer (Kodak Dr78) and then fixed and washed using the recommended procedure. Initial photographic enlargements of the plates were made using a Durst 1000 condenser enlarger and 20 to $25 \mathrm{~cm}$ rapidoprint paper which was processed on an AgfaGevaert rapid print processor. Areas of interest were then magnified either on the Durst enlarger or under the microscope.

The hearts were then examined macroscopically and by conventional histological techniques using haematoxylin and eosin, elastic van Gieson, and Mallory's trichrome stains.

\section{Results}

Studies were made on 18 human hearts. The 9 male and 9 female patients had an age range of 21 to 75 years. The weight of the hearts varied from 175 to 6ro with a median of $330 \mathrm{~g}$. Details of age, sex, heart weight, grade of atheroma, and cause of death are given in the Table. The pattern of the main extramural coronary veins was similar to that described by previous authors (Baroldi and Scomazzoni, 1967; James, 196r; Hood, 1968). In all hearts the coronary sinus drained the great cardiac, left posterior, and left marginal veins, while in 4 it also drained the small and anterior cardiac veins. Filling of the intramural veins of the left ventricular free wall was obtained in all hearts and the pattern of these vessels was distinctive and contrasted with the arterial vasculature in comparable hearts. Twelve hearts, with weights from I75 to $355 \mathrm{~g}$, were from patients who had died of non-coronary causes. Their coronary arteries showed only minimal or early atheroma and there was no evidence of myocardial damage. In these hearts the density of the veins after injection by radio-opaque medium was considerable as illustrated in Fig. I, a microradiograph of a $5 \mathrm{~mm}$ transverse ventricular slice of a $310 \mathrm{~g}$ heart from a 62-year-old man who died

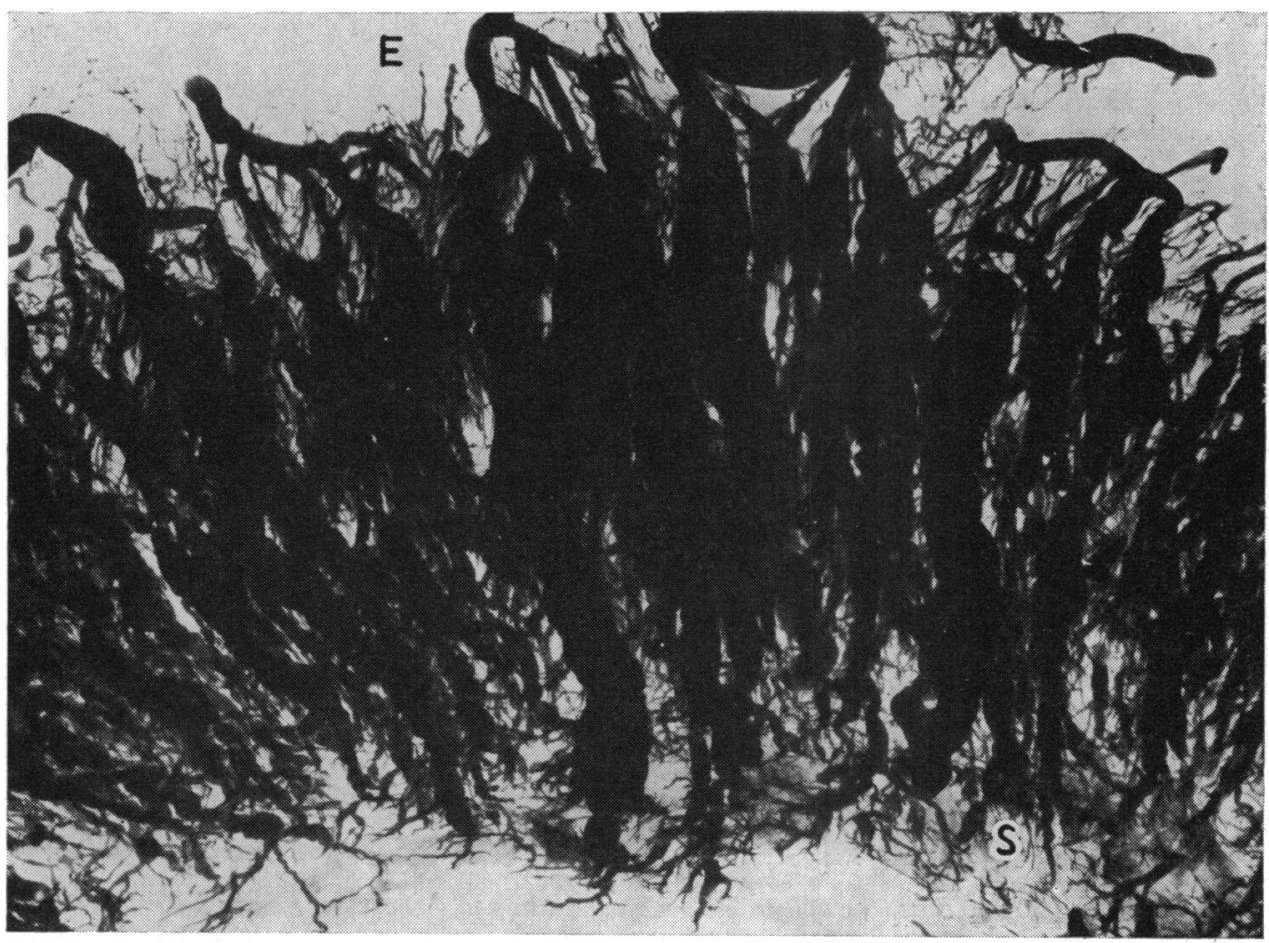

FIG. $2 A$ close-up of the posterior wall of the left ventricle of an adjacent slice to that illustrated in Fig. I. Large drainage veins originate in the subendocardial $(S)$ zone and course fairly directly to the epicardial $(E)$ surface. $(\times 4$. 
from non-cardiac causes. Higher magnification of the myocardial veins of this heart showed large drainage vessels (Fig. 2) beginning in the subendocardial zone and running a comparatively straight course towards the pericardial surface. The calibre of these veins was fairly constant until the subpericardial zone where in small groups they converged to form a single vessel which then entered, almost at right angles, the large pericardial veins. The density of these myocardial drainage veins varied slightly between each 'normal' heart and a less dense pattern is shown in Fig. 3, a microradiograph of a $350 \mathrm{~g}$ normal heart, from a 29-year-old man who died of multiple injuries.

In addition to these large vessels, smaller drainage veins were seen beginning in the middle of the wall and joining the main myocardial veins just before they entered the pericardial veins (Fig. 3). In the subpericardial zone the pattern varied slightly with the small veins coursing directly out to enter the pericardial veins (Fig. 4). Throughout the whole width of the wall the small veins appeared to enter the large drainage veins without an extensive intermediate network of medium sized vessels. The venous pattern within the papillary muscles was also distinctive, with small veins draining the periphery and converging towards the middle into larger vessels.

The left half of the interventricular septum showed a similar venous pattern to the free wall of the left ventricle. Fig. 5 illustrates the veins in the adjacent posterior wall and left side of the posterior half of the interventricular septum. More anteriorly in the midsection of the interventricular septum the appearances were seen to be even more similar, with the main drainage veins (Fig. 6) coursing directly from the subepicardial zone to the large central drainage veins, running alongside the main septal arteries, near the middle of the septum.

In this preliminary study 6 hearts were con-

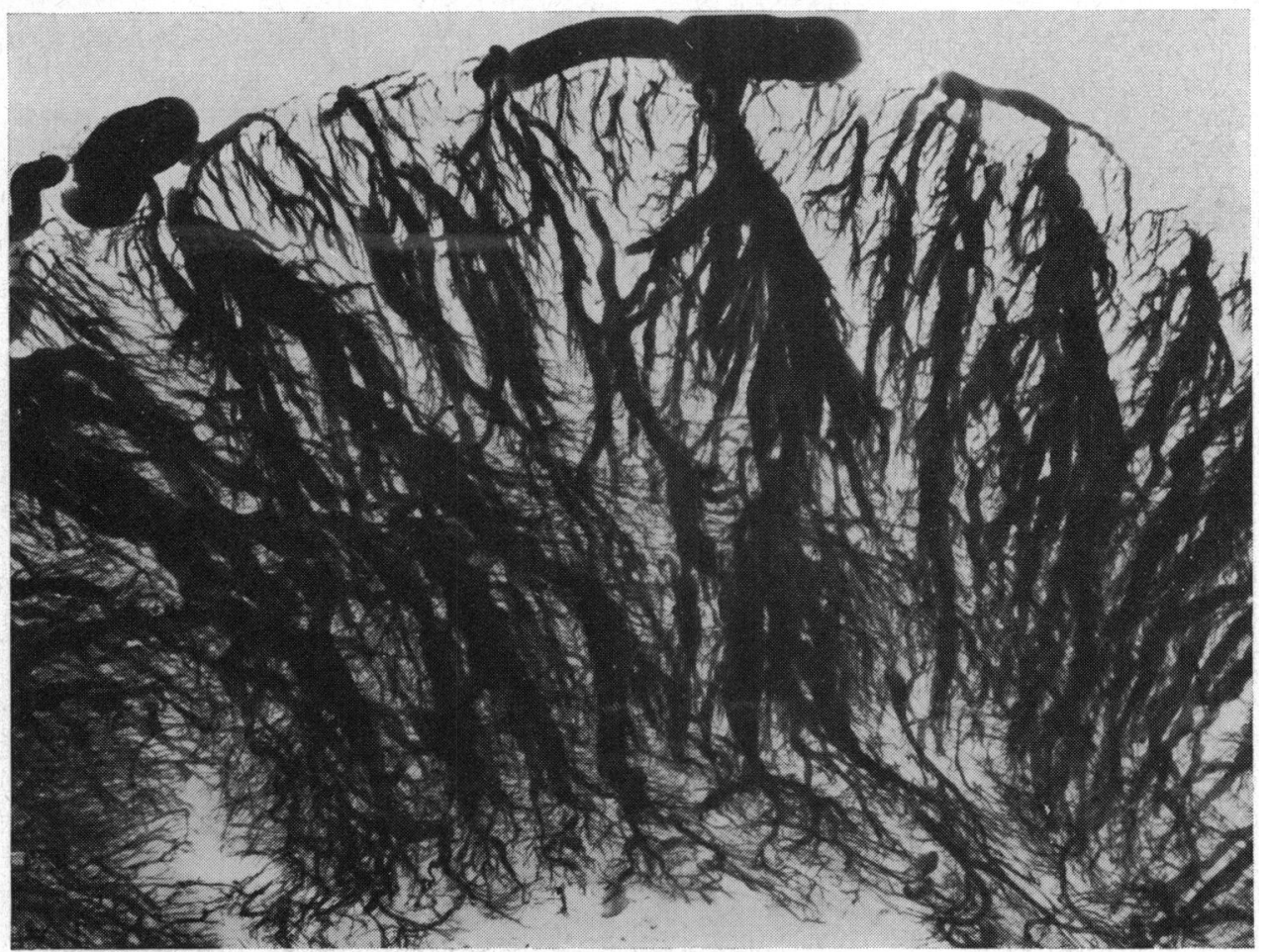

FIG. 3 A similar microradiograph to Fig. 2 but from a $330 \mathrm{~g}$ 'normal' heart of a 29-year-old man who died of multiple injuries. The large myocardial drainage veins are slightly more spread out, while the converging of a small number of these veins to form a single vessel in the outer part of the wall is clearly seen. Drainage veins begin in the middle of the wall as well as in the subendocardial zone. $(\times 4$. 


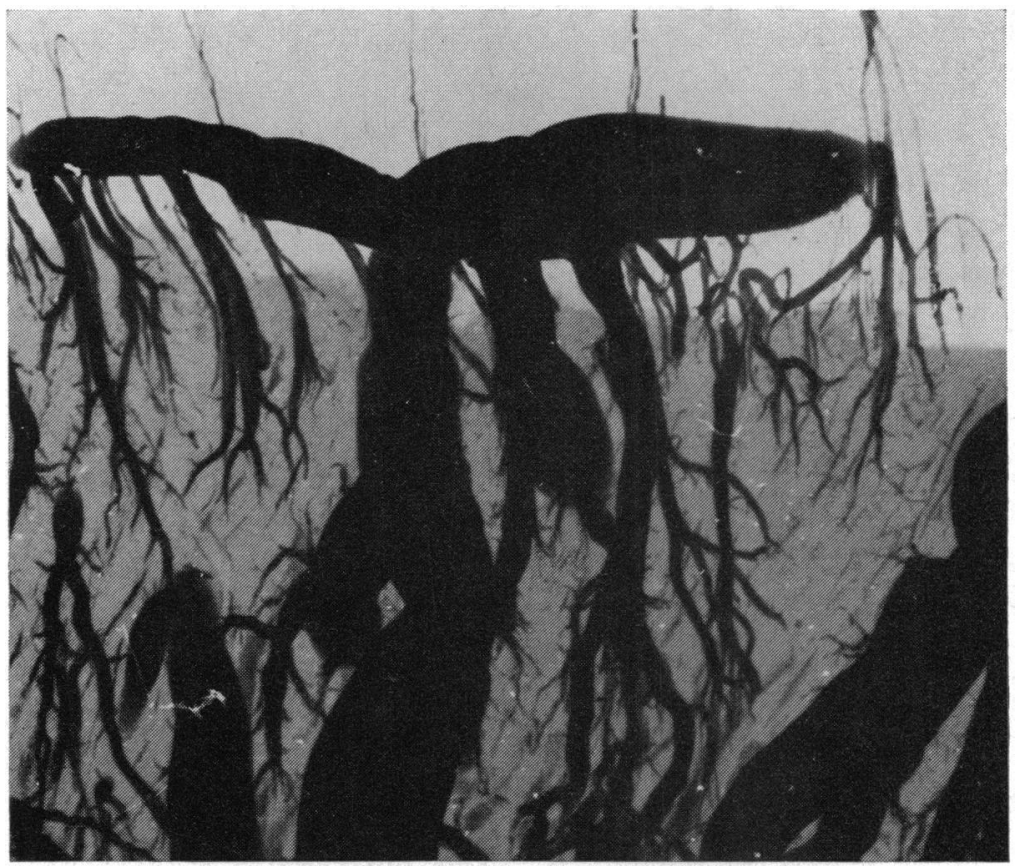

FIG. 4 Close-up of the outer myocardium of a 'normal' heart with small veins, on each side of a large drainage vein, passing straight into a pericardial vein. $(\times 10$.

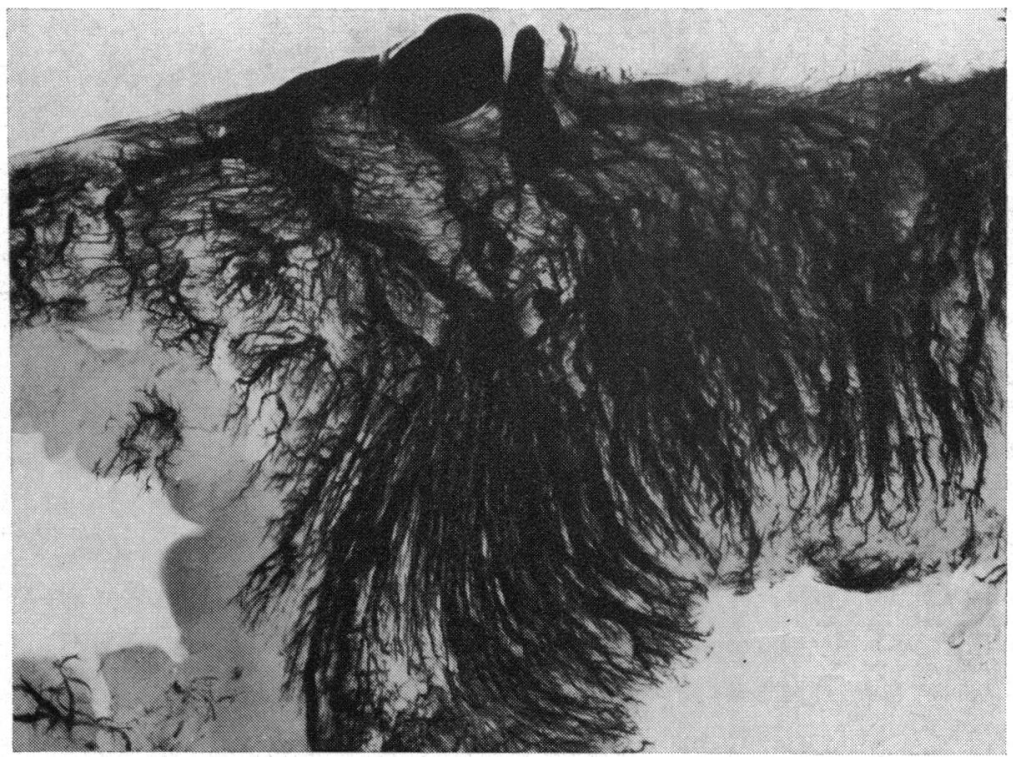

FIG. 5 Microradiograph showing the similarity in vein pattern in the left half of the posterior part of the interventricular septum and the posterior wall of the left ventricle. $(\times 3.5$. 
90 Farrer-Brown and Tarbit

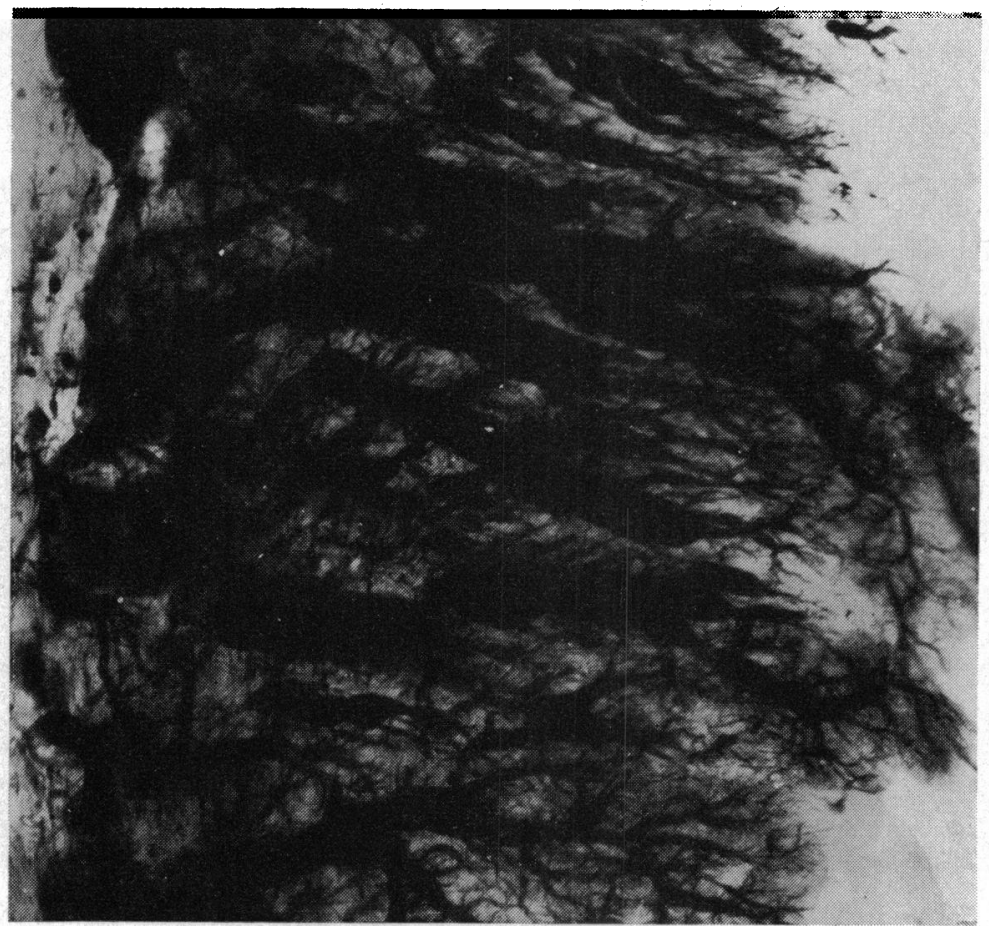

FIG. 6 Large veins draining the left half of the interventricular septum with a pattern very similar to that seen in the free wall of the left ventricle. ( $X$ I2.)

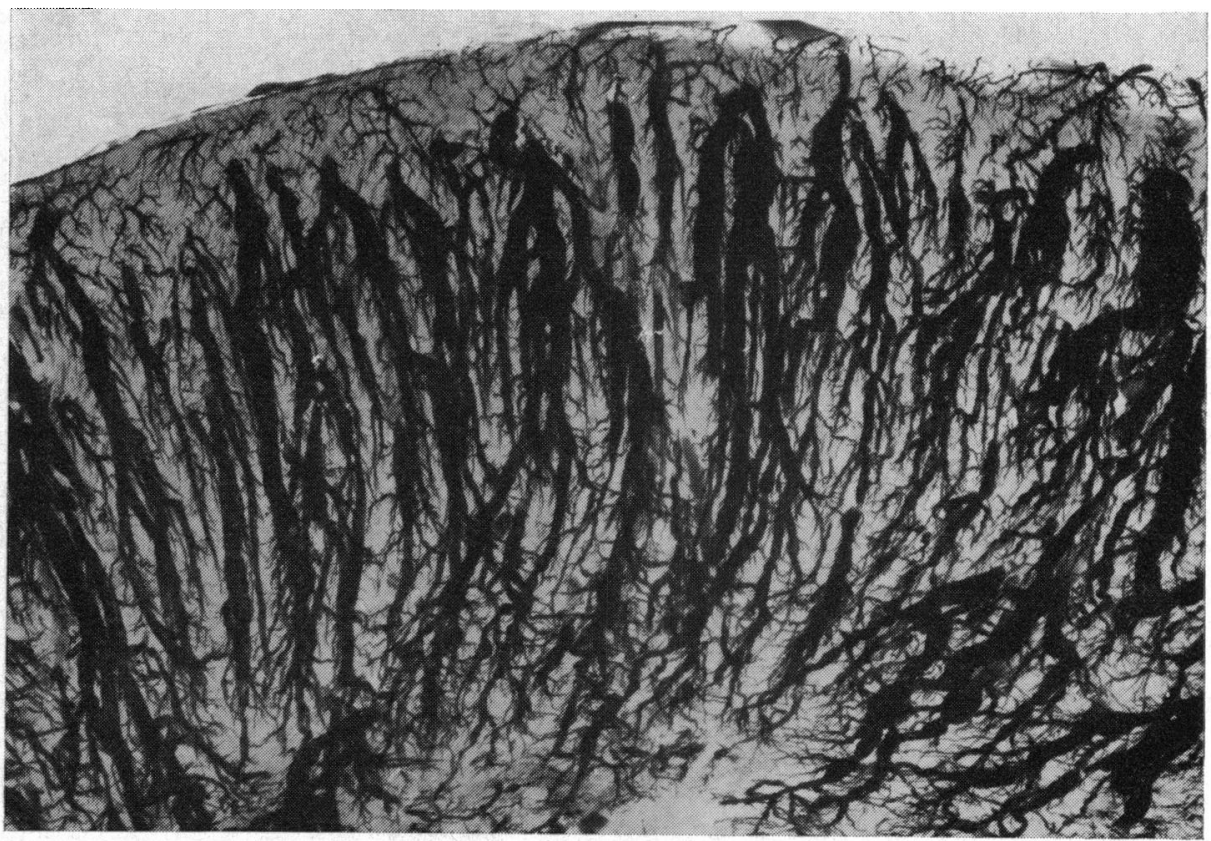

FIG. 7 In left ventricular hypertrophy the drainage veins appear more spread out compared with normal. $(\times 4$. 


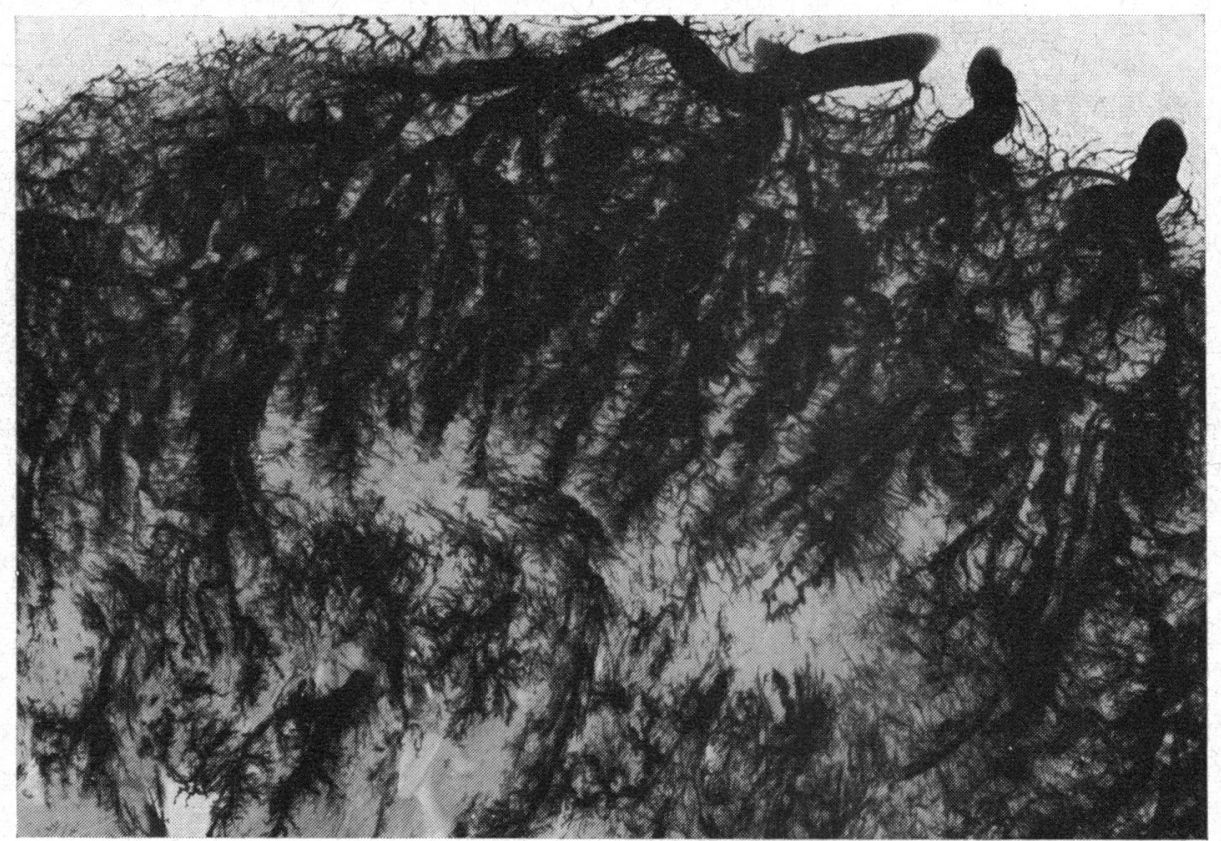

FIG. 8 Areas of fibrosis in the inner myocardium with interruption of the main drainage veins and loss of normal vein pattern. ( $\times 4$.

sidered abnormal, 4 having left ventricular hypertrophy resulting from benign hypertension. The pattern of veins in the hypertrophied myocardium was basically normal though the vessels were more widely separated and consequently overall less dense on $x$-ray (Fig. 7). Two of these hearts, plus 2 other hearts, showed coronary artery atheroma of grade 2 or 3 severity (World Health Organization, 1958). Within small areas of fibrosis of the myocardium of these hearts, there was interruption of the main drainage veins (Fig. 8) and loss of normal pattern. In I, with an old occlusion of the left anterior descending coronary artery and subendocardial fibrosis of the left ventricular free wall, there was absence of main drainage veins in the inner half to two-thirds of the wall and replacement by an abnormal plexus of small veins (Fig. 9). Vessels were seen to run circumferentially (Fig. ro), with a few coursing radially. The appearances suggested that this was the venous component of the 'plexus' of vessels seen following coronary artery injections in hearts with this pattern of disease (Fulton, 1965; Farrer-Brown, 1968c).

\section{Discussion}

The technique previously used by the authors to study the coronary arteries proved readily adaptable for the investigation of the venous system. Leakage around the cannula inserted into the coronary sinus may occur but can be prevented by using a curved surgical needle to insert two sutures around the cannula about $\mathrm{I} \mathrm{cm}$ apart. The ideal pressure needed to inject the veins is debatable, but the range chosen was found to be the best in order to obtain a satisfactory filling of the smaller veins without either causing leakage of vessels or obvious distension of veins on histological sections.

The retrograde filling of the small veins throughout the left ventricular wall and the majority of the interventricular septum after a coronary sinus injection is similar to the finding of Hood (1968), who concluded that the coronary sinus drained 96 per cent of small veins in these areas. However, Hood (1968) described the small vessels as only draining the outer two-thirds of the myocardium and did not demonstrate small veins throughout the wall of the left ventricle.

The present detailed study of a small number of hearts has shown that the pattern of intramural veins differs from the arterial pattern. In agreement with Truex and Angulo (1952), who found on whole heart $x$-rays that the left ventricle contained more venous than arterial radicles, in the present study the former vessels were more dense through- 


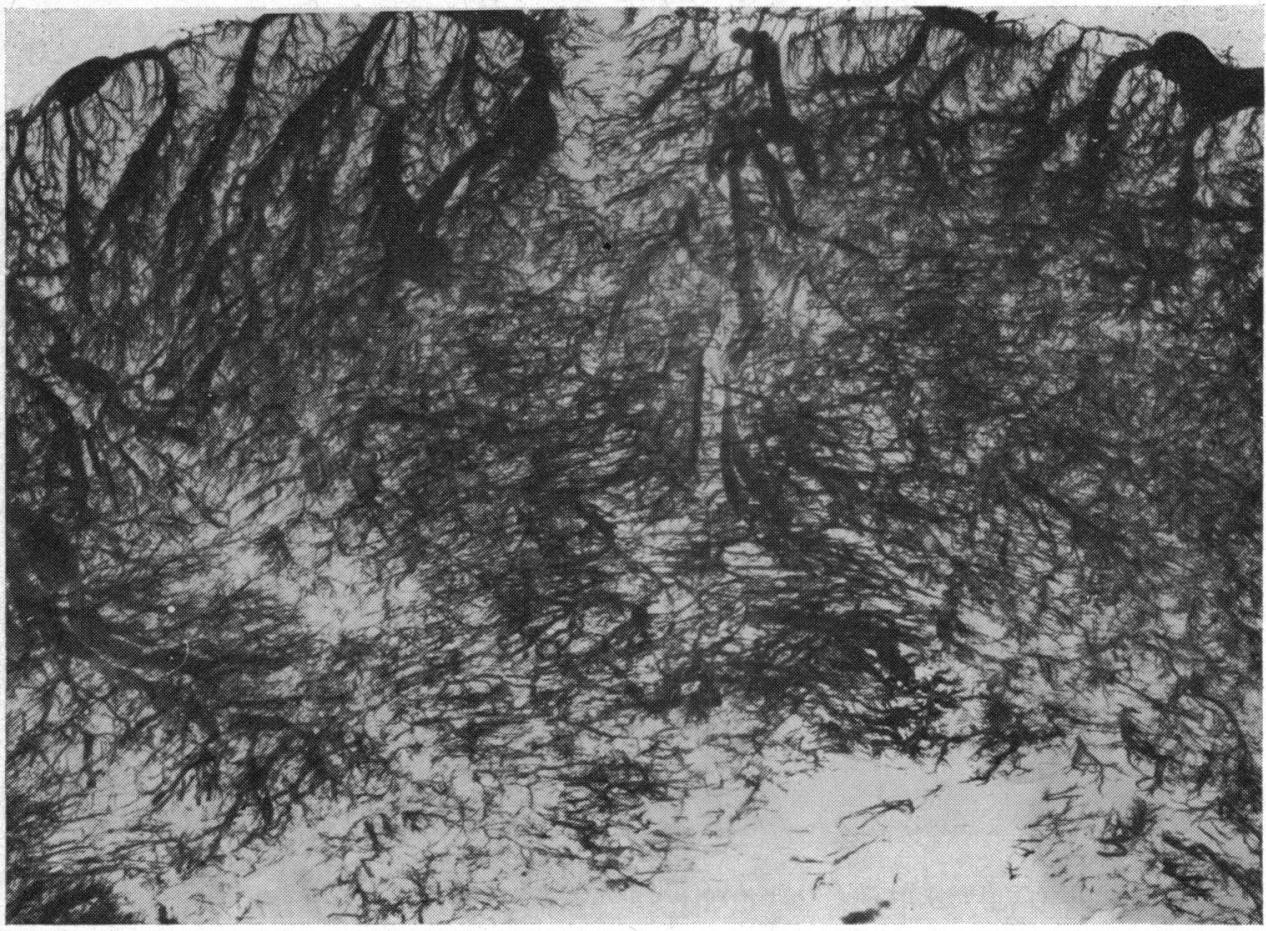

FIG. 9 Microradiograph of the anterior wall of the left ventricle of a heart with generalized severe coronary artery disease. Associated with a laminar type of inner wall fibrosis, there is replacement of the normal vein pattern by a plexus of veins coursing mainly circumferentially, but also radially. $(\times 4$.

out the whole width of the left ventricular wall. In addition, their distribution did not mimic the branching pattern of the majority of myocardial arteries. Instead, drainage veins began close to the endocardium and in the middle of the wall, and coursed fairly directly towards the epicardium maintaining a comparatively even calibre throughout. In general, small veins entered directly into large drainage vessels without any extensive intermediate medium-sized vein network.

The vein pattern in the left ventricle suggests that in the normal heart there is rapid drainage of blood from the myocardium on contraction of the ventricle. There may be a need for rapid flow not only to ensure adequate circulation but also to allow efficient contraction of the myocardium. As there do not appear to be any valves within the intramyocardial veins, muscle contraction, plus a vis a tergo, must be the two most important factors determining the rate of drainage of myocardial blood.

With sudden coronary artery occlusion, stasis of blood in the veins may be important in determining the rate of failure of contractility of the myocardium if the flow and pressure of blood from adjacent collateral vessels are insufficient to maintain an adequate supply. The importance of a diminishing blood supply to the myocardium in hearts with coronary artery disease is well recognized but the possible added significance of an interrupted venous drainage has received little attention.

Once myocardial fibrosis has occurred the venous drainage is altered, with the large venous myocardial veins usually being interrupted in the subendocardial zone. In hearts with a 'plexus' of vessels in the inner half of the wall, found in generalized severe coronary artery disease, blood in the subendocardial zone may have to course circumferentially before reaching a large drainage vein. It is possible that each contraction of the heart may be of insufficient duration to ensure complete drainage of blood via this circuitous pathway. The resulting stasis or oscillation of blood in the partially fibrosed subendocardial zone, already receiving a diminished 


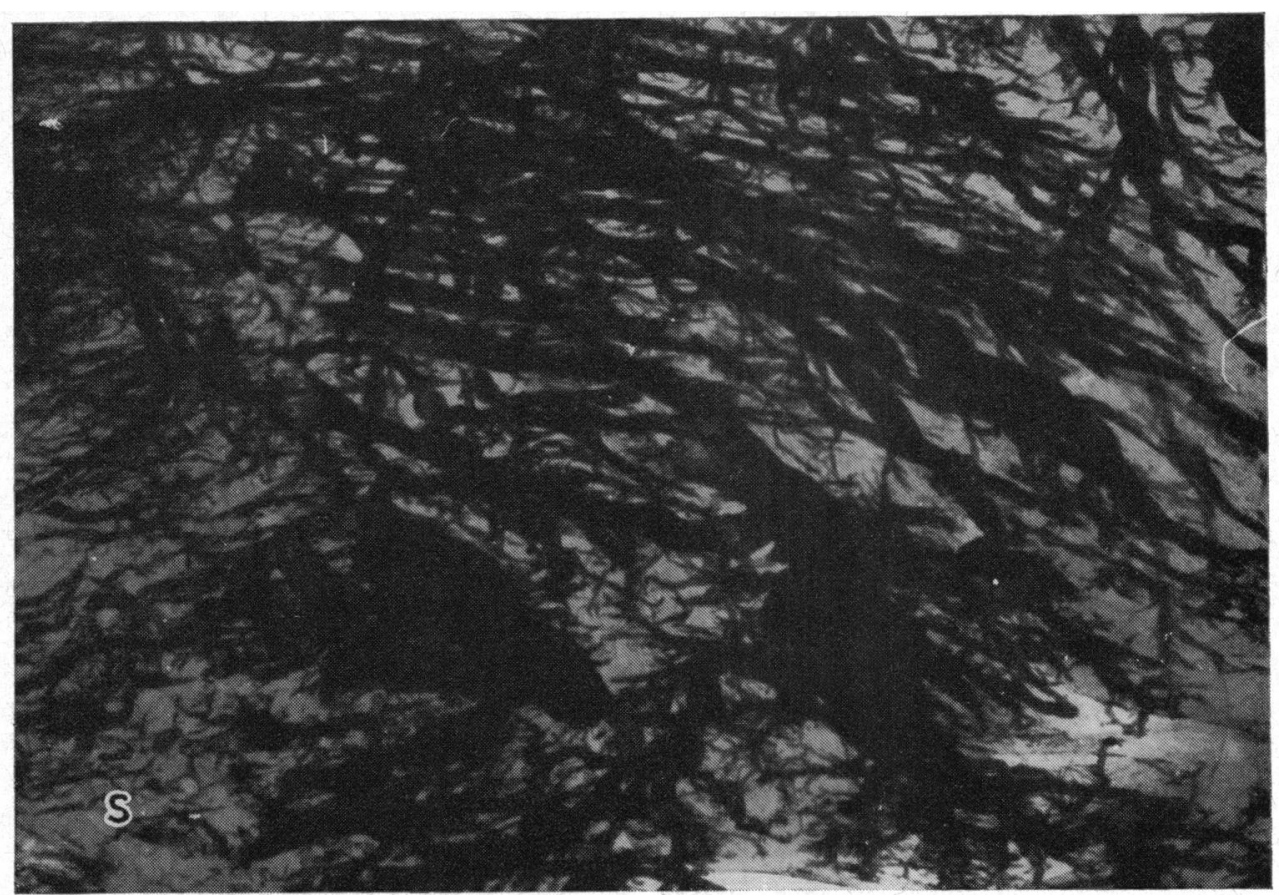

FIG. Io Higher magnification of the circumferential coursing veins comprising the plexus of veins illustrated in Fig. 9 (subendocardium $=S)$. $(\times 17$.

blood supply because of arterial disease, could be significant in the causation or persistence of local ischaemia.

It is suggested that in future physiological and pathological studies evaluating the importance of an adequate arterial blood supply, more widespread consideration should be given to the total blood flow within both the arterial and venous systems.

\section{References}

Baroldi, G., and Scomazzoni, G. (1967). Coronary Circulation in the Normal and the Pathologic Heart. Armed Forces Institute of Pathology, Washington DC.

Farrer-Brown, G. (1967). The Vascular Supply of the Myocardium of the Ventricles of the Human Heart. M.D. Thesis, Cambridge.

Farrer-Brown, G. (1968a). The injection of capillaries, arterioles, and arteries in the ventricles of the human heart by a radio-opaque medium. Cardiovascular Research, 2, 179.

Farrer-Brown, G. (1968b). Normal and diseased vascular pattern of myocardium of human heart. Normal pattern in the left ventricular free wall. British Heart fournal, 30, 527.

Farrer-Brown, G. (1968c). Normal and diseased vascular pattern of myocardium of human heart. Pattern seen with fibrosis of the left ventricular free wall. British Heart Fournal, 30, 537.

Farrer-Brown, G. (1968d). Vascular pattern of myocardium of right ventricle of human heart. British Heart fournal, 30, 679.

Farrer-Brown, G., and Rowles, P. M. (1969). Vascular supply of interventricular septum of human heart. British Heart fournal, 31, 727.

Farrer-Brown, G., and Tarbit, M. H. (1973a). The vascular pattern of the left ventricle of normal hearts from East Africans in Uganda. African fournal of Medical Sciences, 4 , 419.

Farrer-Brown, G., and Tarbit, M. H. (I973b). The vascular pattern of the myocardium of the right ventricle and interventricular septum of normal hearts of East Africans in Uganda. African Fournal of Medical Sciences, 4, 435.

Fulton, W. F. M. (1965). The Coronary Arteries. Charles C. Thomas, Springfield, Illinois.

Hood, W. B. (1968). Regional venous drainage of the human heart. British Heart fournal, 30, ro5.

James, T. N. (196I). Anatomy of Coronary Arteries. Paul B. Hoeber, New York.

Parsonnet, V. (1953). The anatomy of the veins of the human heart with special reference to normal anastomotic channels. Fournal of the Medical Society of New Fersey, 50, 446.

Truex, R. C., and Angulo, A. W. (I952). Comparative study of the arterial and venous systems of the ventricular myocardium with special reference to the coronary sinus. Anatomical Record, 113, 467.

World Health Organization Report of a Study Group (1958). Classification of atherosclerotic lesions. World Health Organization. Technical Report Series, I43.

Requests for reprints to Dr. Geoffrey Farrer-Brown, Bland-Sutton Institute of Pathology, Middlesex Hospital Medical School, London WIN 8AA. 